
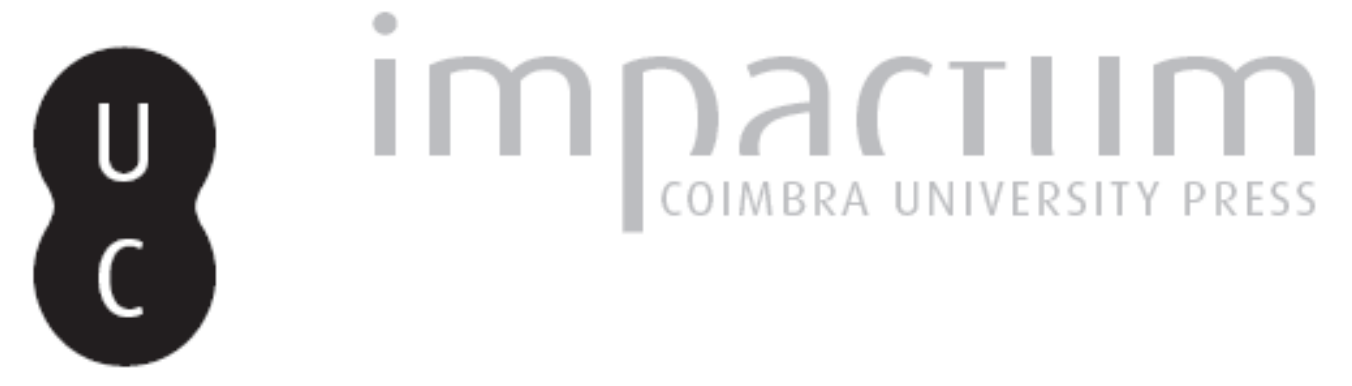

Jóvenes y educadores experimentan a paz viajando por el mar

Autor(es): Guetta, Silvia

Publicado por: Imprensa da Universidade de Coimbra

URL persistente:

URI:http://hdl.handle.net/10316.2/36834

DOI:

DOI:http://dx.doi.org/10.14195/1647-8622_14_8

Accessed : $\quad$ 26-Apr-2023 13:21:02

A navegação consulta e descarregamento dos títulos inseridos nas Bibliotecas Digitais UC Digitalis, UC Pombalina e UC Impactum, pressupõem a aceitação plena e sem reservas dos Termos e Condições de Uso destas Bibliotecas Digitais, disponíveis em https://digitalis.uc.pt/pt-pt/termos.

Conforme exposto nos referidos Termos e Condições de Uso, o descarregamento de títulos de acesso restrito requer uma licença válida de autorização devendo o utilizador aceder ao(s) documento(s) a partir de um endereço de IP da instituição detentora da supramencionada licença.

Ao utilizador é apenas permitido o descarregamento para uso pessoal, pelo que o emprego do(s) título(s) descarregado(s) para outro fim, designadamente comercial, carece de autorização do respetivo autor ou editor da obra.

Na medida em que todas as obras da UC Digitalis se encontram protegidas pelo Código do Direito de Autor e Direitos Conexos e demais legislação aplicável, toda a cópia, parcial ou total, deste documento, nos casos em que é legalmente admitida, deverá conter ou fazer-se acompanhar por este aviso. 


\section{educação e democracia}

ESTUD OSD OSÉCULO

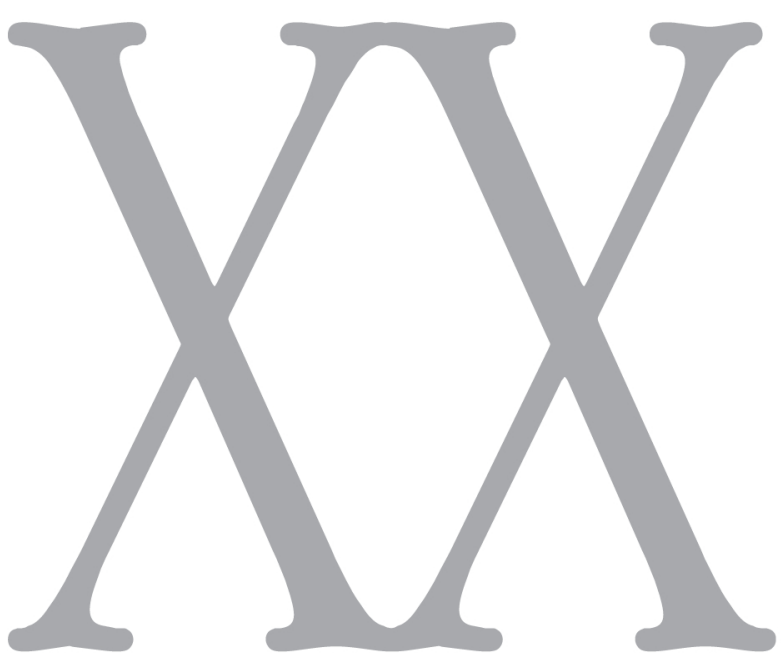

número 14 • 2014

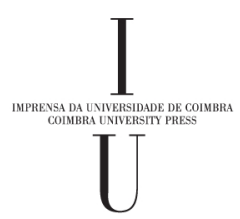




\section{JÓVENES Y EDUCADORES EXPERIMENTAN}

A PAZ VIAJANDO POR EL MAR*

Silvia Guetta 
Silvia Guetta, Doutorada em “Teoria e História do Processo Formativo”. Professora do Departamento de Educación y Psicología da Universidad de Florencia, Itália. Lecciona e investiga nos domínios da Pedagogia Social da Pedagogia Intercultural, da Educação para a Paz. Coordena a Cátedra UNESCO "Sviluppo Umano e Cultura di Pace".

* Tradução do texto de italiano para espanhol: Andrés Palma Valenzuela. Revisão do texto: Maria Helena Damião. 


\section{Introducción}

Este trabajo se centra en el análisis de la contribución de Rami Andrei Rodan ${ }^{1}$ al desarrollo de la cultura de paz mediante un innovador y estimulante proyecto que tuvo por escenario el medio ambiente natural del mar. Rami amaba el mar como una realidad que le atraía desde niño. La sentía familiar y la vivía como un entorno natural que encendió en él los más bellos sentimientos y las mas sugestivas imaginaciones. El mar era para él un amigo con quien hablar, un espacio donde encontrarse a sí mismo así como un lugar del que extraer energías positivas y profundas. Un entorno natural que le ofrecía enormes posibilidades de intercambio y conocimientos sin los límites impuestos por las fronteras. Es difícil saber las maravillas que Rami oyó y vio en su relación con el mar y con todos los mares del Mediterráneo.

El encuentro con los saberes marinos fue una realidad madurada en el tiempo aunque realizada relativamente tarde porque la primera profesión de Rami fue la de director de cine y espectáculos. En realidad poseía un «ojo» especialmente sensible para asumir las expresiones en sus diversas formas de comunicar, de expresarse a sí mismas y de ocultar sus propias necesidades. Con particular hondura y experiencia captaba lo que la naturaleza era capaz de comunicar, al tiempo que podía transmitir, conocimientos y saberes importantes para compartir la paz y superar los obstáculos y adversidades del conflicto.

Sentía con fuerza las inquietudes, sufrimientos y el peso del conflicto palestinoisraelí; el sinsentido de los dolores y los miedos, lo absurdo de la negación de los derechos humanos, la necesidad de abrirse a los demás para encontrarse, conocerse y construir juntos algo nuevo. Pero no era suficiente pensar el lugar y el tema de la paz. Porque las cosas pueden cambiar, pensaba Rami, para posibilitar una transformación, es necesario que todo vaya unido a un renovado compromiso educativo. De ahi la idea de pensar en el proyecto Sails Paz (Velas de la Paz) referido a dos grupos-objetivo: educadores/constructores de paz y adolescentes/jóvenes, con la intención de llevar la propuesta a la Cátedra transdisciplinar de la UNESCO «Desarrollo Humano y Cultura Paz» de la Universidad de Florencia.

Desde tales antecedentes y perspectivas, este autor se movilizó, junto con otros socios relacionados con el «Centro Peres para la Paz», para la constitución de la ONG, "Velas Paz», cuyo objetivo era promover experiencias educativas en diferentes contextos sociales a partir del poder terapéutico, estimulante y beneficioso del mar. A través de experiencias en el mar y con el mar, esta ONG pretendió crear las condiciones

\footnotetext{
${ }^{1}$ Rami Andrei, fallecido en 2012, nació en Rumanía pero se trasladó en su infancia a Israel donde se dedicó durante más de treinta años al cine y a la televisión en calidad de productor, director, docente y artista, obteniendo dentro y fuera de Israel diversos reconocimientos por sus producciones. Durante los últimos años de su vida ideo un proyecto de Educación para la Paz destinado a facilitar el proceso de reconciliación entre israelíes y palestinos, denominado Sails for Peace, al tiempo que se centró en el desarrollo de procesos de rehabilitación e inclusión a través del conocimiento y la relación con el mar.
} 
necesarias para apoyar a la mujer y su recuperación del estrés post-traumático, la reinserción social y el potencial de las personas con discapacidad. El reto de Rami era aunar, de forma integral y creativa, las virtudes y cualidades de los viajes en vela y las habilidades náuticas y marítimas con las aspiraciones humanas por la paz, la justicia social, la igualdad de oportunidades y los derechos humanos.

Viajar juntos es una buena manera de conocerse. Ir en un barco que surca el mar constituye un bello modo de crear cooperación e intercambio, reflexionar sobre la belleza de la naturaleza, así como una forma ideal para aprender otros lenguajes: estar bien con uno mismo y estar bien con los demás. Compartir un viaje de este tipo requiere la aceptación emocional de los demás, al margen de su realidad u origen social y cultural, el desarrollo personal de sus posibilidades y la capacidad de asumir la responsabilidad de sus acciones, tanto frente así mismos como ante los demás.

Vivir la experiencia del mar se convierte por ello en una importante ayuda a las personas para aprender sobre sí mismos, descubrir sus fortalezas y talentos ocultos y comprender el valor y el significado del trabajo de equipo. Por tanto, es fácil pensar que este empeño formativo pueda experimentarse, tanto en el dar como en el recibir, como un cambio de vida positivo.

Tomadas como punto de partida estas referencias relativas a la misión y razones que impulsaron la creación y el trabajo de la ONG Sails Paz, pensamos que la labor efectuada por Rami, prematuramente interrumpida, sigue siendo un mensaje de paz y amor, transportado por los vientos, las corrientes y las olas del mar capaz de tocar los corazones y las mentes de las jóvenes generaciones.

El proyecto se ha desarrollado despues con la Catedra Transdisciplinar UNESCO «Desarrollo Humano y Cultura Paz» y ello fue tanto para el director, Prof. Paul Goldsmith, como para el resto del grupo, una experiencia única y desafiante en la investigación y diseño de temas de convivencia pacífica, en la problemática de los conflictos en Oriente Medio y en el desarrollo de la Cultura Paz. La construcción compartida de la idea del proyecto que aquí se presenta, fue también la base de otras ideas y propuestas de acción. En el trabajo para el desarrollo de la cultura de la paz, implementado durante los últimos años mediante la programación y diseño de vías de búsqueda e intervención, la Catedra Transdisciplinar se comprometi a ofrecer apoyo pedagógico, teórico y metodológico y a garantizar la coherencia del diseño reconociendo el valor, el significado, el potencial y la originalidad del proyecto presentado por Rami. Y eso también fue lo sucedido con una experiencia piloto del proyecto, cuando el equipo de investigación organizó en los mares de Tel Aviv «Un fin de semana de la vela y de la Paz», en el que participaron nińos, nińas y educadores israelíes y palestinos junto a la Catedra Transdisciplinar UNESCO de Florencia. 


\section{Un proyecto original}

El proyecto empezó a definirse a inicios del año 2007 bajo el nombre de Sails for Peace $^{2}$. Quiso ser una iniciativa estimulante e innovadora para educar a los jóvenes acerca de la convivencia pacífica, en un entorno natural como el del mar, que buscó dirigirse a un amplio espectro de beneficiarios. La idea y objetivos del proyecto surgieron de la hoda convicción de sus socios israelíes y palestinos de que es posible trabajar juntos por la paz empeñados en el desarrolo de experiencias educativas transformadoras capaces de deconstruir las representaciones psico-socio-culturales del conflicto y de la cultura de guerra con el fin de construir una nueva concepción de la convivencia humana no dependiente ni justificadora de la presencia del enemigo, el peligro y el miedo.

Su objetivo consistió en involucrar a jóvenes, educadores, maestros, voluntarios y estudiantes israelíes, palestinos e italianos en una experiencia de viaje en barco de vela en que la singladura se convirtiese en un encuentro consigo mismo y con los demás. En una experiencia interpersonal a través del cual fuera posible analizarse y llegar a conocerse unos a otros descubriendo a la vez la riqueza del conocimiento del mar y los beneficios de la convivencia pacífica.

Se diseñó el proyecto con el fin primordial de crear una estrategia capaz de limitar, reducir y gestionar el nivel de hostilidad y desconfianza mutua crecido en los años de conflicto entre jóvenes palestinos e israelíes, contando con al apoyo neutral de jóvenes italianos, de 17 a 21 años de edad, que podían ofrecer diferentes modelos de relación y solución de los conflictos. Las acciones se estructuraron para poder realizar juntos algunos días de viaje, concretando e incluyendo procesos que ayudasen a ser capaces de pensar, sentir y poder vivir juntos más allá de los prejuicios y la hostilidad mutua que la cultura y la educación han generado a menudo mediante la ética de la guerra. El proyecto buscó formar en las relaciones pacíficas a través de la experiencia directa de las necesidades de cooperación e intercambio; aspectos inherentes a la formación de un espíritu de paz y necesarios para todo proceso de reconciliación ${ }^{3}$.

\subsection{Algunos aspectos del proyecto}

Sus áreas de interés: el mar Mediterráneo ha sido considerado como área de investigación y lugar donde llevar a cabo la iniciativa. Se consideró un espacio privilegiado para la experiencia pues en el proyecto la referencia a la relación entre mar, tierra, costas y puertos, es esencial para explorar una de las oportunidades que ofrece la navegación: la construcción de saberes interculturales.

\footnotetext{
${ }^{2}$ Con los años esta original iniciativa formativa fue en parte integrada y adaptada a las necesidades de las propuestas a las que se ha dirigido. Aquí se presenta esencialmente la estructura básica del proyecto.

${ }^{3} \mathrm{La}$ investigación y el diseño se formalizó mediante un acuerdo entre la ONG Peace Sails y la Cattedra Transdisciplinare UNESCO (Ver Orefice, 2010).
} 
Su organización: El núcleo central de la organización del proyecto estaba formado por la ONG Peace Sails y la Catedra Transdisciplinar UNESCO de la Universidad de Florencia. Sobre esta base como punto de partida, el proyecto se basó en la estructura de una asociación integrada por un socio palestino y un representante del área territorial italiana. A tal estructura se añadieron centros de investigación, organizaciones no gubernamentales locales y universidades israelíes.

$\mathrm{Su}$ temporalización: El proyecto fue diseñado para un período de tres ańos. Durante este tiempo se organizó una primera fase de encuentro e intercambio de conocimientos entre los socios para la puesta en común del proyecto y un programa formativo para aprender y experimentar la metodología prevista (investigación-acción participativa, aprendizaje cooperativo, educación al aire libre) para profundizar en el conocimiento de los aspectos teóricos y problemáticos de la Educación para la Paz, diseñar los instrumentos para la aplicación, dirección y evaluación del proyecto. La segunda fase consisitó en el inicio de la experiencia en el barco, contando sólo con una formación básica previa para navegar, el inicio de los intercambios en una plataforma de cooperación para promover la breve experiencia de navegación durante el verano y la participación de un grupo, en esencia intercultural, de jóvenes y adultos.

El proyecto como respuesta a un contexto de conflicto: Las discusiones políticas sobre la paz en Oriente Medio hacen evidente de forma inmediata el amplio espectro de opiniones sobre cuestiones específicas que requiere el camino de los acuerdos, formales y políticos y la necesidad de identificar vías alternativas para promover un cambio radical, reconociendo las razones que originaron el fracaso de iniciativas anteriores. Para las partes en conflicto el concepto de Paz es muy sentido pero semántica y conceptualmente se considera de modo diferente. Ello se evidencia también en la presencia de numerosas organizaciones y actividades que trabajan en el campo de los temas de la paz, los derechos humanos y la cooperación. A pesar de los grandes pasos dados durante los últimos ańos para encontrarse y dialogar con el mundo de la sociedad civil, en los que ha desempeńado un trabajo capilar y extenso la labor de las ONG y las asociaciones para hacer emerger la cuestión en el ámbito de la educación formal, el tema ha sido con frecuencia evitado.

El objetivo general del proyecto: Es la creación de un grupo creciente de jóvenes, profesores y educadores, palestinos, israelíes y europeos que, de forma conjunta y cooperativa, son capaces de investigar sobre la paz en rutas de interés común y son capaces de difundir los conocimientos adquiridos contribuyendo personal y creativamente al desarrollo humano de la comunidade local. Estos son sus temas y objetivos específicos:

- Preparar, elaborar e implemetar un perfil claro de experto en Educación para la Paz con competencias sobre el mar y habilidades de navegación y, a la vez, sobre educación activa y democrática de jóvenes, profesores e instructores y los principios de la coexistencia pacífica.

- Crear una red de socios y medios de comunicación locales para desarrollar proyectos destinados al desarrollo de una cultura de paz creativa y participativa. 
- Comprender la naturaleza, orígenes y efectos de la violencia sobre los jóvenes y su educación.

- Dotar a los jóvenes y adultos de habilidades para la resolución de conflictos personales y sociales ayudándoles a comunicarse de forma no violenta.

- Fomentar la busqueda de instrumentos de comunicación no violenta y desarrollar un compromiso concreto con la no violencia.

- Reforzar la creencia de que el cambio y mejora social se activan en primer lugar, a partir de lo que pensamos, sentimos y hacemos los individuos y grupos.

- Desarrollar habilidades de comunicación activa, entendimiento y compasión e inculcar el principio de respeto a los demás, los derechos humanos y la cooperación.

- Desarrollar la capacidad de "pensar de manera crítica y constructiva" para investigar de forma reflexiva sobre las creencias personales y colectivas, crear dudas acerca de los modelos socio-educativos actuales y superar el escepticismo que bloquea las iniciativas de paz.

- Desarrollar habilidades empatico-afectivas hacia las diferentes percepciones de la realidad y las aspiraciones, deseos y expectativas de los demás.

- Involucrar a los participantes en el conocimiento del mar, la navegación y la convivencia en el barco, el reconocimiento de las peculiaridades, sus puntos fuertes, sus energías, sus desafíos y su potencial de crecimiento y cambio personal.

- Estimular e inspirar a los participantes en el amor al mar, la navegación y la riqueza de la vida en el barco.

- Comprender el potencial que ofrece la naturaleza del mar, aprender a reconocer y beneficiarse de ello tanto a nivel individual como en grupo.

- Desarrollar la autoestima en base a los resultados obtenidos mediante la adquisición de nuevos conocimientos y habilidades de navegación, gestión de tareas y el arte de la navegación.

- Enfatizar y desarrollar caminos de "Educación para la paz con la experiencia" como un proceso cultural y social en el que los grupos en conflicto, así como terceros, puedan confronatrse y cumplir tareas y desafíos comunes.

- Elaborar manuales educativos y de experiencia marítima sobre "Educación para la Paz con experiencia" mediante el desarrollo de estrategias y prácticas interculturales y de paz.

- Crear un grupo creciente de educadores y artistas capaces de enseñar la paz como valor permanente y eterno; establecer alianzas mediterráneas e internacionales para el desarrollo de proyectos internacionales fuera de la región interesada; formar embajadores de la paz encargados de la difusión del conocimiento de la paz en su entorno, capaces de trabajar para ampliar los sitios y canales de paz a través de la participación inclusiva y local; contactar y difundir información en los medios de comunicación para cambiar y mejorar la opinión pública local, palestina, israelí e internacional. 
- Ayudar a cambiar el ambiente "sin esperanza de paz en nuestras vidas", que sigue creciendo en la región y promover un clima de esperanza, cambio y aceptación mutua.

- Crear crear un clima de legitimidad de las iniciativas de paz con formas diversas y variadas.

Nacieron algunas ONGs israelíes y palestinas, sobre todo tras los intentos de acuerdos de paz de Oslo de 1993, que abrieron caminos de relación mutua gracias tambien a la ayuda de la Unión Europea y a través de los centros «Peres Center for Peace» (Israel) y «Panorama Center» (Palestina) que dieron lugar, a pesar de reflejar las diferentes sensibilidades políticas de ambos partidos, al inicio de grandes momentos de encuentro y planificación compartida. Fueron ocasiones importantes para el conocimiento reciproco, el nacimiento de nuevas relaciones humanas y el descubrimiento de la necesidad de tener que poner todo en acción para lograr resultados positivos. Asimismo deben ser consideradas, en sus respectivos contextos, las diferentes percepciones sobre la paz y los derechos humanos, la violencia y la justicia social, con el fin de evitar edificar sobre frágiles fundamentos un nuevo sistema de derechos humanos, autonomía y justicia social.

No hay duda de que las actitudes hacia la paz pueden expresar paradigmas sociales y culturales muy diversos, sobre todo, en una región como Medio Oriente que ve los diferentes patrones de violencia entre israelíes y palestinos exprersarse de muchas maneras causando dificultad y confusión respecto a las posibles direcciones que el "proceso de paz» puede tomar.

La retirada de Israel de la Franja de Gaza en 2005 y la victoria de Hamas en las elecciones palestinas de 2007 son algunos de los acontecimientos recientes más importantes en este escenario político. La dificultad de los líderes políticos israelíes y palestinos para llegar a una solución diplomática puso en evidencia la importancia de crear una cultura de la paz entre las personas afectadas. Dentro de Israel, por ejemplo, hay actitudes muy diferentes respecto a la paz caracteristicas de las diversas subpoblaciones existentes (judio/árabe, religiosos/no religiosos; sionista/no sionista; asquenazí/sefardíes, izquierda/derecha, centro/periferia, hombre/mujer, drusos, circasianos, beduinos, etc.). Similar variedad de puntos de vista existe tambien en contexto palestino, donde las distintas religiones (cristiana/musulmana), la procedencia (Gaza/Cisjordania), las posiciones políticas, las cuestiones de género o las condiciones sociales, crean visiones e hipótesis de solución de los problemas muy diversas.

En el plano educativo también debe tenerse en cuenta que las barreras y separaciones existentes entre las dos realidades ha aumentado en los últimos años el desconocimiento mutuo entre los estudiantes y jóvenes israelíes y palestinos. Saben muy poco del otro y lo ven casi siempre como enemigo o entidad hostil y negativa. A pesar de las exhortaciones de las organizaciones no gubernamentales, los dos sistemas educativos/escolares han trabajado mucho en estos aspectos. Las diversas experiencias adquiridas en la educación no formal han demostrado tambien que, para enseñar a 
trabajar la paz de forma constructiva, es necesario que exista entre los jovenes la conciencia de que, en el otro lado, es posible encontrar interlocutores disponibles, que caminan y afrontan los temas de la convivencia con similares motivaciones y en la dirección de la tolerancia activa y la paz participada.

La claridad y la conciencia de los valores que las sociedades transmiten a las generaciones futuras lleva a considerar que se ha de cambiar la dirección y el modo de presentar el tema, para superar estereotipos y prejuicios sobre el otro, visto en este caso, como el enemigo. Si bien es cierto que la paz la firman los estadistas, la educación y la formación, en sus diferentes modalidades, sobre todo formal, tienen responsabilidades específicas en la transmisión de los valores y los conocimientos. Estos, usando continuamente, símbolos y contenidos de los valores de la cultura de la guerra, son también, de forma implícita, transmisores y perpetuadores de los conflictos armados.

La educación tiene una gran responsabilidad en el desarrollo de habilidades concretas y operativas para la realización de la paz, la apertura del camino hacia la reconciliación y la transformación de los conocimientos locales, la difusión de las experiencias de la ciudadanía activa, participativa y creativa, y en la asunción e implementación, por parte de todos, del respeto a los derechos humanos y a la protección del medio ambiente.

Como ya se indicó, en el proceso de Paz de Oslo de 1993, el tema fue bien planteado al constatarse la necesidad de la creación de agentes de Paz para ambos pueblos (IPCRI, 2008). Lo que no ha funcionado, a pesar de ello, ha sido la falta de comprensión del significado del precio de la paz o la participación requerida por ambas partes para lograr un acuerdo. Los profundos sentimientos de hostilidad no han hallado el modo de transformarse y la dimensión del sentimiento, aún poco considerada en los contextos de resolución de conflictos, ha continuado generando miedo, inseguridad y falta de confianza. ¿Hasta cuando la cultura del cambio y la cooperación se sentirá abrumada por la cerrazón y el antagonismo/abuso?. El conflicto de Oriente Medio muestra con claridad la necesidad de compartir propósitos y metas y de crear condiciones operativas concretas para que profesores, educadores y trabajadores sociales pertenecientes a todas las partes involucradas, abran un debate, mediante un trabajo crítico y reflexivo sobre sus experiencias, representaciones y expectativas. También es posible pensar que, a causa de los diferentes climas políticos, estas prácticas pueden realizarse y llevarse a cabo en lugares neutrales y lejanos al conflicto.

El proyecto considera la presencia de la ira, la desesperación, el miedo y la inseguridad como fenómenos arraigados de generación en generación durante los últimos setenta años. Un ethos de la guerra presente en las diferentes formas de educación y en la construcción de los saberes y el conocimiento de las personas y las instituciones de las dos sociedades. No obstante, el trabajo sobre la paz desde la óptica pedagogico-educativa ha sido en muchos aspectos hostigado, especialmente mediante vías formales referidas a leyes presentes en las diferentes realidades (Salem \& Kaufman, 2007). 
También debe tenerse en cuenta cómo durante los últimos años se han logrado acuerdos de paz, primero, entre Egipto e Israel y, despues, con Jordania; a pesar de que se han puesto en marcha acuerdos entre autoridades israelíes y palestinas, la violencia, el dolor, la preocupación y las dificultades han seguido alimentando el ethos de la de la guerra, la percepción de la imposible solución del conflicto y la falta de confianza en las instituciones y sus representantes. Las personas ya no creen que sea posible hacer algo por la paz y toda tentativa se considera desde el principio como un fracaso. En tal contexto se extiende además el extremismo religioso a causa de la pobreza, la pérdida de la esperanza y los contextos de fragilidad en que se encuentran las personas. La necesidad y la búsqueda de seguridad llevaron a la segregación total entre las dos poblaciones porque vallas y separaciones, construidas para un mejor control del peligro de atentados, han impedido a la gente verse y encontrase. Desafortunadamente esto se convirtió también en un terreno fértil donde han crecido nuevas formas de racismo, xenofobia, fanatismo y terrorismo.

Por fin, y dentro de esta síntesis de la situación, es importante indicar cómo el proyecto busca ubicarse en el interior de una serie de nuevas iniciativas para la paz al tiempo que pretende ofrecer un camino alternativo y novedoso de encuentro, cambio y transformación a pesar de que aún sean muy fuertes y este muy arraigadas, en ambas partes, variadas formas de escepticismo y oposición a los cambios que pueden llevar a la convivencia pacífica entre los diferentes grupos.

\subsection{Su integración con la Educación para la Paz}

La educación para la paz que aquí consideramos trata de provocar en los participantes la necesidad de hablar y discutir los problemas concretos y cotidianos en los que se encuentran implicados. Ello ayuda a entender que, además de buscar soluciones que en muchos casos son similares, aunque los grupos parezcan vivir experiencias opuestas y no compartibles, es necesario considerar qué valores pertenecen a esas respuestas. De este modo, el proyecto sitúa de inmediato a los niños y educadores ante hechos concretos y ello permite activar una forma rápida de «aprender haciendo». Tal práctica facilita un aprendizaje que crea las bases para la adquisición de los valores de la cultura de paz, para cambiar las actitudes de hostilidad en los países de acogida y de escucha activa, para reconocer la importancia de comprender sus propios sentimientos y reconocer sus comportamientos con el fin de vivir juntos de forma constructiva. Mediante el proyecto «Velas por la Paz» los participantes experimentan la paz de forma directa y participativa junto con el significado y el empeño que requieren los valores y las posibles acciones de tolerancia, cooperación, pluralismo, gestión de conflictos y respeto de las necesidades humanas, los derechos y la naturaleza.

El proyecto tiene como objetivo concluir que el medio ambiente que preparamos y construimos y los espacios donde vivimos son responsables tambien de la construcción de una cultura de paz. Por ello se optó por un entorno natural, muy dinámico y rico en conocimientos, que puede ser peligroso pero, a la vez, extremadamente útil y divertido. 
Un ambiente que aún requiere protección, cuidado y respeto, al igual que las personas. En tal sentido, la Educación para la Paz asume también un referente intercultural específico: el lenguaje de la naturaleza, así como el del mar, abierto a la necesidad de relacionarse con experiencias culturales diferentes y múltiples. Si todo ello se integra y convierte en un instrumento que permite la comunicación entre mundos diversos, e incluso hostiles, como es el caso de los jóvenes israelíes y palestinos, entonces la experiencia se vuelve aún más profunda y compleja. Crear un entorno compartido con chicos que pertenecen a diferentes culturas, prepararlos para un periodo de convivencia en un ambiente que requiere el compromiso y la participación de todos, no es simple. Se necesita voluntad y disponibilidad, así como una inversión consciente de energías y habilidades para la vida. Para llevar a cabo una experiencia de convivencia pacífica e intercultural se han identificado diferentes enfoques interculturales. Comenzamos por la Outdoor education (educación al aire libre) y el Joint Maritime $e^{4}$ (Comisión paritaria marítima).

Se entiende por educación al aire libre, el aprendizaje que tiene lugar fuera del ambiente cerrrado del aula escolar y, en este caso, se adapta a este tipo de intervención por la forma en que facilita el contacto con el entorno natural. Tal enfoque no se propone como una simple educación en el medio, sino que centra su atención en la integración de tres aspectos: el descubrimiento del conocimiento del medio ambiente, la aventura y el proceso de aprendizaje (Higgins \& Nicol, 2002). Estas condiciones de aprendizaje, estimulante y rico, permiten a los estudiantes no vincularse a las costumbres y normas sociales que plantean obstáculos a los cambios y experiencias. Las experiencias de educación al aire libre son diferentes entre sí porque cada una de ellas pone en juego expectativas y deseos, competencias y habilidades diversas. Cada una se vincula a diferentes teorías de referencia al tiempo que se encuentra influida por el modelo de relación con el entorno que los educadores proponen. Es mas, la tipología ambiental elegida, con sus posibilidades de movimiento y recursos, hace que cada encuentro sea una aventura en sí misma. Este aprendizaje fuera del aula no sustituye el marco formal, más bien lo complementa, fortalece y estimula desde la búsqueda de experiencias de aprendizaje gratificantes. Este tipo aprendizaje exterior es importante tanto para alcanzar los objetivos educativos, instrumentos y técnicas utilizadas como por el desarrollo gradual de las habilidades de los participantes.

Gracias a la educación al aire libre la relación educativa se gestiona de manera diferente. La relación entre profesores/educadores y estudiantes cambia con respecto a la clase, la aventura es compartida por todos los participantes que participan de la experiencia inclusiva de sentirse parte de un grupo que vive la misma experiencia. Las reglas formales que distancian la relación con el adulto desaparecen, convirtiéndose en comportamientos de ayuda y la comprensión mutua donde la mente, el cuerpo y las

\footnotetext{
${ }^{4}$ La Comisión Paritaria Marítima (CPM) es un órgano permanente bipartito que asesora al Consejo de Administración sobre cuestiones marítimas, incluyendo el establecimiento de normas para la industria del transporte marítimo internacional.
} 
habilidades crean la comunicación en el grupo. Estas experiencias también conducen a procesos de reflexión donde, a veces, es necesario deconstruir modelos y presentaciones personales y sociales, con el fin de adquirir nuevas competencias y abrirse a cuestiones y preguntas novedosas que buscan respuestas, incluso, fuera de los límites establecidos por los cinco sentidos (Higgins \& Nicol, 2002)

La educación al aire libre se relaciona con modelos teóricos como el constructivismo, la formación experiencial, los procesos de desarrollo en los ambientes de aprendizaje, la metacognición, la inteligencia múltiple y otros diversos estilos de aprendizaje (Gilbertson, 2006). Salir fuera de los esquemas y entornos facilita romper las barreras que separan a las personas, crean indiferencia y alimentan los prejuicios. Las experiencias al aire libre pueden ayudar a superar juntos todas las dificultades y los peligros, al margen de diferencias de religión, cultura, género o capacidad. Una experiencia de educación al aire libre en el mar ayuda asimismo a crear espíritu de grupo y de trabajo en equipo y a gestionar y fortalecer habilidades sociales y cognitivas previamente aprendidas.

El mar, como un espacio natural y emocionante de cambio, es un buen catalizador para los encuentros. Para los jóvenes, éste se presenta como un intrumento único que propicia la reflexión sobre los elementos culturales y sociales que influyen en el comportamiento de los grupos y que, en las activiades al aire libre, pierden significado invirtiendo mas bien su experiencia y competencia de cambio, participación, intercambio y cooperación para lograr resultados compartidos. El barco es una pequeńa habitación que restringe los movimientos y desplazamientos. Cada persona debe saber cómo moverse en relación con el movimiento y el espacio de los demás. Así se expresan las obligaciones y necesidades, incluidas las tareas y roles de los que cada persona debe asumir desde su responsabilidad. Cada participante es importante, no es superfluo ni marginal.

Todos participan en el bienestar del entorno en que las personas viven en ese momento, con las posibilidades y el conocimiento personal. Viajar juntos algunos días lleva a todos a compartir la gestión diaria y, al mismo tiempo, a conocer, compartir y cambiar el fondo cultural de pertenencia. El momento de la comida, por el hecho de que sea auto gestionado, es un espacio para compartir y desarrollar intercambios para conocer modelos de comportamiento, hábitos y gustos diversos.

Para muchas personas, el mar es un contexto familiar y conocido pero para otros puede ser una fuente de ansiedad, preocupación, dificultades de gestión y de percepción de inseguridad. Emociones que pueden llegar a ser objeto de reflexión y conexión con los frecuentes y diarios temores y preocupaciones que la presencia de conflictos genera y mantiene. El mar también ofrece una sensación de libertad de espacio, de movimiento y de sorpresa. Para los jóvenes ello es importante pues ayuda a entender las contradicciones que a veces generan los conflictos internos. Encontrar el modo de resolverlos es una experiencia importante en el proceso personal de búsqueda y para la resolución de conflictos. La navegación a lugares lejanos permite además conocer a personas que viven en diferentes países y de manera diversa. Esto ayuda a los jóvenes a ampliar la perspectiva y la percepción de la realidad, tanto de forma individual como en 
grupo al tiempo que a crear un espíritu viajero responsable que ayuda a adquirir habilidades para reconocer y respetar las diferencias.

La referencia al Joint Maritime es útil para entender, no tanto la necesidad de construir un sistema de seguridad y defensa de las costas, sino para adquirir los conocimientos y habilidades para "explotar" la energía positiva del mar y lograr una buena navegación. El control activo de la situación es una de las competencias marítimas más importantes pues cada acción debe proyectarse de acuerdo con lo que dice el mar que pide a sus navegantes preparación, flexibilidad, auto-sostenibilidad y movilidad. Por todo ello el mar debe ser definido no como un lugar de conflicto sino como medio de vida importante para la supervivencia de todos los seres vivos y el equilibrio del medio ambiente. Una defensa en sentido planetario y no local que se concreta en la sostenibilidad de las acciones que se llevan a cabo en el mar.

\subsection{Su significado, metodología y finalidad}

Los principios educativos que apoyan el proyecto «Velas por la Paz» son fruto de una integración de los aspectos teóricos, los valores de la Educación para la Paz, los métodos y los objetivos pedagógicos de la Educación al aire libre que mejor pueden responder a las exigencias formativas de los jóvenes palestinos, israelíes e italianos y del modelo de construcción del conocimiento de la Investigación-Acción-Participativa, que sigue el proceso natural de aprendizaje. La necesidad de los palestinos de adquirir experiencia con el mar se considera un recurso importante para la denominada «Educación para la Paz con la experiencia». Los socios palestinos e israelíes de «Velas por la Paz» estan firmemente convencidos de que uno de los métodos más importantes y eficaces para promover la Educación para la Paz puede ser eficaz gracias a su acción concreta, primero, en un ambiente neutral y, luego, en las vidas concretas de los jóvenes.

A través de la experiencia directa, la paz no es sólo un concepto social o político, sino la forma real y concreta que hace avanzar y madurar el potencial humano a través de un proceso de interacción de las diferencias, haciendo un uso óptimo y motivante de los puntos de fuerza y las capacidades de cada participante. La creencia es que la Educación par la Paz, realizada a través de la experiencia concreta, es un camino fundamental y necesario en un momento en que, por ambas partes, se sigue apoyando la idea de que las soluciones al conflicto se puede lograr mediante la imposición de la fuerza y la violencia. Por todo ello la Educación para la Paz a través de la experiencia y la participación constructiva de los jóvenes debe ser aún más promovida por las organizaciones de la sociedad civil y locales al tener éstas un papel clave en el diseño de este tipo de iniciativas en sus territorios.

Lamentablemente, las autoridades locales y la asociaciones sociales no han sabido responder de forma adecuada a estas demandas. Los escépticos creen que la Educación para la Paz no ayuda a resolver los problemas de inmediato porque las situaciones son muy difíciles y complicadas y a veces parece imposible la paz en la región palestino-israelí. Los 
cínicos rechazan por su parte la idea argumentando que lo que se hace es demasiado poco o demasiado tarde. Frente a ellos, los defensores del proyecto «Velas por la paz» creen, a diferencia de quienes intentan mantener el statu quo, que la propuesta de modelo de Educación para la Paz puede tener un efecto positivo y transformador en los jóvenes que participan en esta iniciativa de formación y que, con el tiempo, afectará a la realización de la verdadera paz en las sucesivas fases de reconciliación.

La metodología propuesta para el proyecto es bastante compleja debido a la necesidad de querer integrar diferentes enfoques útiles para la realización de las actividades educativas programadas. Esta opción metodológica que integra tanto la investigación como la formación es la Investigación-Acción-Participativa (IPA). El modelo de referencia es el propuesto por la Catedra Transdisciplinar UNESCO como un enfoque participativo, integrado y democrático para el desarrollo del pensamiento y el sentimiento que se encuentra en relación con los estudios, investigaciones y experiencias dirigidas en este campo por Paul Orefice (2006). La base de esta propuesta metodológica es la convicción de que el enfoque de aprendizaje propuesto a los sujetos debe ser coherente con la manera natural en que tiene lugar el aprendizaje. En la práctica en esta metodología, "la operación de reproducción, es parte del proceso y está dentro de ella hasta dar espacio a su potencial para el desarrollo humano" (Orefice, 2006, vol I, p. 11). En otras palabras, aún lo que es adecuado para el aprendizaje informal, la problemática, las motivaciones y acciones concretas, debe encontrar su propio espacio en una iniciativa consistente de aprendizaje intencional. En el proyecto se intenta la experimentación metodológica como una síntesis entre la IPA, la Educación al aire libre y colaborativa y ciertos aspectos del aprendizaje cooperativo.

Debido a su caracter flexible y a su capacidad para responder a diferentes preguntas y cuestiones del medio ambiente no sólo humanas, la metodología de la IPA se adapta fácilmente a los diferentes contextos y situaciones. Uno de los aspectos fundamentales de este curso es acercar y establecer contactos directos entre los investigadores y el público interesado en la solución de los problemas que les afectan directamente, incluyendo la metodología, que incorpora el enfoque de la investigación científica y la vida cotidiana de las personas. La manera de trabajar juntos para buscar la forma de resolver cuestiones y problemas se situa, considerado la experiencia de educación al aire libre, en un plano simétrico.

Todos los implicados están en el mismo nivel, porque están directamente involucrados, según su posición y punto de vista, en las actividades de investigación, consideradas como una valiosa herramienta para el cambio social. La investigación y la acción trabajan juntas para la tranformación y se alimentan y reclaman recíprocamente. La dimensión participativa se refiere en particular a la participación social y emocional que entra en juego en el aprendizaje. Dimensión que se ve en la lógica de sentirse totalmente integrada con la del pensar y el hacer. La participación permite que exista participación de la persona que comparte el deseo del conocimiento y la consiguiente 
disponibilidad de generar nuevos aprendizajes. Por ello, la participación es un sentimiento común que es posible adquirir compartiendo lo que se ha aprendido.

\section{Conclusiones}

Como conlusión de lo anterior, se puede afirmar que proyectos como éste ayudan a compreder de que forma los jóvenes tienen la necesidad de experimentar de manera directa tanto su propia dimensión humana como la de los demas al tiempo que la de llevar a cabo experiencias concretas apoyadas sobre la confianza en sus propios recursos y capacidades. Asimismo entendemos que la realidad de cada persona puede cambiar si se utilizan diferentes perspectivas de observación y relación con los demás y con el medio ambiente.

Para todos aquellos que han participado en el proyecto, el mar ha sido una gran oportunidad para lograr una nueva percepción de la realidad. Éste se ha convertido sin duda en un espacio importante, lejos del conflicto, muy apto para comprender tales significados, en un ámbito libre e intercultural, lleno de nuevos lenguajes capaces de poner a prueba el conocimiento directo de los problemas y, sobre todo, en un espacio de posibilidades de descubrimiento y esperanza.

El proyecto Sails for Peace ha ofrecido en definitiva la posibilidad de experimentar todo esto al tiempo que ha estimulado, a través de la participación de jóvenes y educadores en el mismo, la articulación de nuevos instrumentos educativos para lograr la consolidación de la paz. 


\section{REFERENCIAS BIBLIOGRÁFICAS}

GILBERTSON, K.; BATES, T.; McLAUGHIN, T; EWERT, A. - Outdoor Education, methods and strategies. USA: Human Kinetics, 2006, 224 p. ISBN 13: 978-0736047098 224.

HIGGINS, P. \& NICOL, R. - Outdoor education, authentic learning in the context of landscapes. Sweden: Kisa, 2002, 943 p. ISBN 91-631-2904-3,

IPCRI (Israel/Palestine Center for Research and Information) - Peace education in Palestine and Israel. Learning about our neighbors, 2008. Disponible en www: <url: http://www.ipcri.org/ files/learning\%20about\% 20the\%20other.pdf>.

OREFICE, Paolo - La ricerca azione partecipativa. Teoria e pratiche. Vol 1. La creazione dei saperi nell'educazione di comunità per sviluppo Napoli, Liguori. Fiorenza: Linguori, 2006, 372 p. ISBN-13: 978-8820738600

SALEM, W. \& KAUFMAN, E. - Proposed guiding principles for Israeli/ Palestinian academic cooperation: Translating the shared adherence to academic freedom into action. Paris: UNESCO, 2007, 34 p. Disponible en www: <url: http://unesdoc.unesco.org/images/0015/001564/ 156437e.pdf>. 